\title{
Patentablauf von Blockbuster-Medikamenten
}

Pirmin Pfister

Keine Interessenverbindungen im Zusammenhang mit diesem Artikel.
In naher Zukunft werden ungewöhnlich viele big selling drugs (z. B. Sortis, Zyprexa, Plavix, Seroquel) ihren Patentschutz verlieren. Was an der Börse als patent cliff bezeichnet wird, kann eine Sparchance für die Gesundheitswesen sein. Ob das schweizerische dieses Sparpotential optimal nutzen kann, hängt vom Verhalten der verschiedenen Entscheidungsträger ab.

\section{Sparpotentiale}

Die Akzeptanz der Generika wird durch das Vertrauen in diese Medikamente beeinflusst. In der NZZ am Sonntag vom 10. Juli 2011 steht in der Überschrift eines Artikels betreffend Generika: «Günstiger, manchmal aber auch weniger gut.» Ein Patient, der nur diese Headline liest, fühlt seine Meinung bestätigt, dass weniger teure Medikamente auch weniger gut seien. Gemäss der Studie von Rebecca L. Waber [1] gibt es nämlich einen Zusammenhang zwischen dem Preis einer Pille und dem Vertrauen in deren Wirksamkeit. So war in dieser Arbeit der Placeboeffekt der teureren Pille grösser als derjenige der kostengünstigeren.

\section{Ob das Sparpotential optimal genutzt werden kann, hängt vom \\ Verhalten der verschiedenen Entscheidungsträger ab.}

Ist das öfters beobachtete Misstrauen in günstige Generika aus evidence-based Sicht gerechtfertigt? Der Harvard-Forscher Kesselheim fand in einer systematischen Analyse von peer-reviewed publications [2], die zwischen 1984 bis 2008 zu diesem Thema erschienen waren, keine Qualitätsvorteile der kardiovaskulären Originalpräparate gegenüber Generika.

Das Forscherteam analysierte zusätzlich noch die im genannten Zeitraum erschienenen Editorials zu diesem Thema. Erstaunlicherweise sprachen sich die Hälfte der Editorial-Autoren gegen die Umstellung von kardiovaskulären Originalpräparaten auf Generika aus. Dies, obwohl es aus evidence-based Sicht keine Gründe für diese Ablehnung gab. Kesselheim et al. sehen eine mögliche Erklärung dieser Diskrepanz darin, dass die Editorial-Autoren durch einzelne, persönliche Erfahrungen oder nicht-klinische Studien beeinflusst worden sein könnten. Zudem wurde auch die Möglichkeit einer Beeinflussung

\section{Expiration du brevet de plusieurs médicaments «blockbusters»}

La plupart du temps, le passage d'un médicament original à un générique se fait sans difficulté, hormis quelques rares cas d'intolérance aux excipients. Une exception cependant: les médicaments dits à dose critique, ou critical dose drugs (principes actifs à marge thérapeutique étroite). Pour ces substances, il convient d'examiner avec la plus grande attention les avantages et les inconvénients de la substitution. De plus, le passage à un générique nécessite une surveillance stricte du patient, ce qui a également un prix - et on peut dès lors se demander si la substitution est réellement économique et appropriée sur le plan médical. Il serait donc essentiel qu'une commission médicale indépendante élabore des directives pour déterminer les cas où ce changement n'est pas approprié.

En Suisse, la part de génériques est plutôt faible, vu les réticences évoquées plus haut. La mesure la plus efficace et la plus simple à mettre en œuvre consisterait à prescrire un principe actif à la sortie de l'hôpital, ce qui permettrait de contrer la réticence du patient à se tourner vers un générique (en cas de remise de préparations originales). Ce n'est qu'en augmentant suffisamment la participation pécuniaire pour les préparations originales que l'on peut amener les patients à choisir un générique (théorie de l'aversion à la perte). La nouvelle réglementation en matière de quote-part pour les médicaments devrait permettre de diminuer les prix. Lors de chaque substitution, il convient de veiller à ce que les charges des cabinets, hôpitaux, caisses-maladie et patients n'augmentent pas de manière excessive, sans quoi les économies réalisées seraient neutralisées.

durch finanzielle Beziehungen mit der Pharmaindustrie angesprochen.
Korrespondenz:

irmin Pfister

Zentrumspraxis

CH-8105 Regensdor

pirmin.pfister[at]gmx.ch 
Auch bei Medikamenten nimmt die Bindung zum Produkt mit der Zeit zu. Die Umstellung auf ein Generikum kann beim Patienten eine Verlustaversion und dadurch Ablehnung hervorrufen.

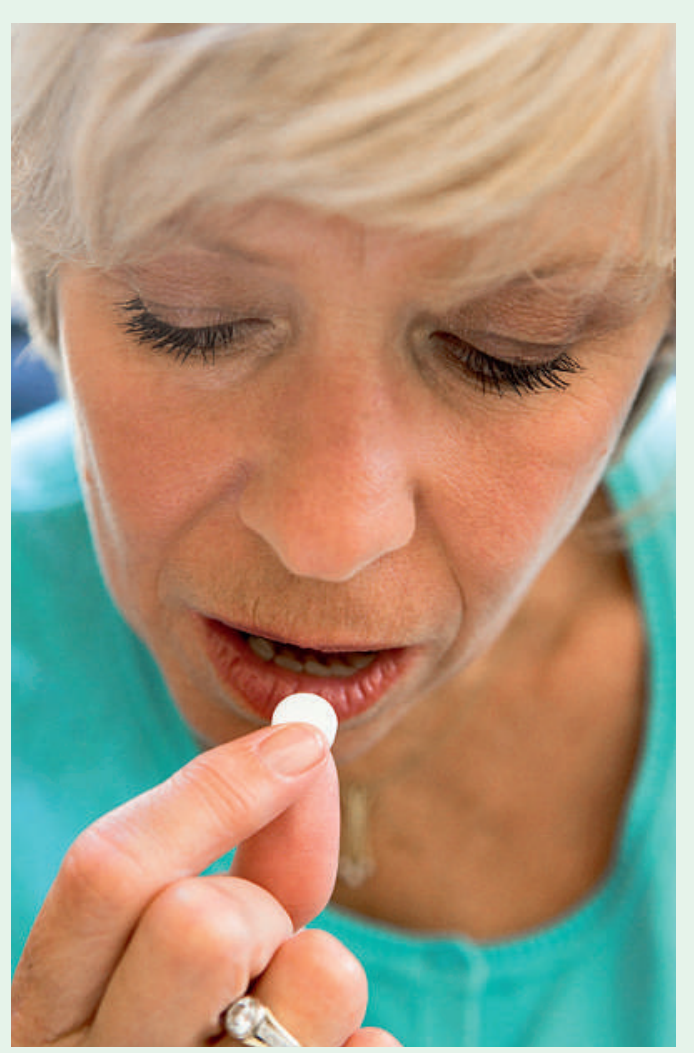

\section{Umstellung auf Generika}

Der obengenannte NZZ-Artikel bezieht sich auf eine Bioequivalenz-Studie [3]. Diese zeigt, dass bei etlichen Generika von Antiepileptika die maximale Wirkstoffkonzentration im Blut um bis zu $25 \%$ von derjenigen des Originals abweicht (die europäische Arzneimittelbehörde EMA erlaubt 20\% Abweichung). Zwischen verschiedenen Generika untereinander war der Unterschied noch grösser. Dies bedeutet jedoch nicht, dass das Generikum weniger gut als das Original ist. Bei Substanzen mit einer engen therapeutischen Breite wie Antiepileptika muss der Patient aber in der Umstellungsphase genau überwacht werden. Dies, um den gewünschten Wirkstoffspiegel im Blut sicherzustellen und um Compliancefehler zu verhindern. Bei jeder Antiepileptikumsub- behalten, wenn sich aufgrund des neu geregelten Medikamentenselbstbehaltes (seit 1.7.2011; resp. ab 1.1.2012) die Frage eines Wechsels von einem Generikum auf ein anderes stellt. Die Neueinstellung direkt auf ein Generikum ist jedoch unproblematisch.

Erschwerend bei der Umstellung vom Original auf das Generikum ist die Verlustaversion des Menschen. Die in der Wirtschaftswissenschaft etablierte Theorie der Verlustaversion wurde vom Nobelpreisträger Daniel Kahneman und seinem Forscherkollegen Amos Tversky beschrieben [5]. Verluste beeinflussen das Verhalten des Menschen weitaus stärker als Gewinne in ähnlicher Grössenordnung. Aufgrund der Verlustaversion neigen Menschen dazu, auch dann an bereits Vorhandenem festzuhalten, wenn es eine bessere Alternative gibt («Status-quo-Effekt»). So wird der Patient nur auf ein Generikum wechseln, wenn der persönlich «gefühlte Gewinn» deutlich grösser als der «gefühlte Verlust» ist. Der «gefühlte Gewinn» kann auch ein niedrigerer persönlich zu leistender Kostenanteil bedeuten.

Die Bindung zum Produkt, das man besitzt, nimmt mit der Zeit zu. Auch scheint es einen Zusammenhang zu geben mit der Situation, in der man erstmals mit dem jeweiligen Medikament ausgestattet wurde. Bekommt der Patient nach einer Hospitalisation bei Austritt ein Rezept mit den Originalpräparaten, dann wird er sich mit den vom Hausarzt angebotenen Generika nur ungern anfreunden. Dies bringt sowohl bei der Umstellung auf Generika als auch betreffend Compliance Probleme. Daher ist es wichtig, dass die Spitalärzte auf dem Rezept in erster Linie den Wirkstoff und eventuell in Klammern noch das im Spital abgegebene Präparat aufschreiben. Die Reihenfolge scheint formell unbedeutend, hat aber eine entscheidende, vertrauensbildende Wirkung auf den Patienten.

2010 belief sich der Anteil der Generika am generikafähigen Markt in der Schweiz auf 48,2\% [6]. Im Vergleich mit anderen Ländern ist dies relativ bescheiden. In Deutschland entfielen beispielsweise im gleichen Jahr 82\% des Umsatzes im generikafähigen Markt auf die Nachahmerprodukte [7]. Könnten die

\section{Es gibt einen Zusammenhang zwischen dem Preis einer Pille und dem Vertrauen in ihre Wirksamkeit.}

stitution (Original auf Generikum; Generikum auf anderes Generikum und sogar Generikum auf Original) steigt nämlich das Risiko eines epileptischen Anfalls [4].

Bei Substanzen mit enger therapeutischer Breite ist auch der Wechsel von einem Generikum auf ein anderes mit Risiken behaftet. Dies gilt es im Auge zu oben beschriebenen Widerstände überwunden werden, würde auch in der Schweiz der Anteil der Generika am generikafähigen Markt steigen.

Wie hoch der Spareffekt beim Ablauf der Patente von Blockbuster-Medikamenten ist, hängt auch vom Preis der Generika ab. In der Schweiz sind die Generika im Schnitt doppelt so teuer wie in anderen Län- 
dern Europas [8]. So wird zum Beispiel in Deutschland 52\% der Schweizer Generikapreise bezahlt, in Grossbritannien 36\%, in den Niederlanden 33\%, in Italien $74 \%$ und in Österreich $69 \%$.

Mit der neuen Regelung des dynamisierten differenzierten Medikamentenselbstbehaltes werden Preissenkungen erwartet. Die Regelung schreibt vor, dass der Patient 20\% Selbstbehalt bezahlen muss, falls der beim Patienten wohl nur über eine noch höhere pekuniäre Eigenleistung bei Originalpräparatkonsum $\mathrm{zu}$ erreichen (Verlustaversion). Mit der neuen Regelung des Medikamentenselbstbehaltes sollen die Preise reduziert werden.

Bei allen Interventionen sollte im Auge behalten werden, dass durch die Neuregelungen nicht grosse Umtriebe bei Arztpraxen, Spitälern, Krankenkassen

\section{Erschwerend bei der Umstellung vom Original auf das Generikum ist die Verlustaversion des Menschen.}

Preis für ein Generikum oder Original über einem Referenzpreis liegt. Für Medikamente innerhalb des Referenzpreises sind lediglich $10 \%$ Selbstbehalt zu entrichten.

\section{Fazit}

Die in der Schweiz erhältlichen Generika haben eine gute Qualität und Wirksamkeit. In der Regel ist ein Wechsel von einem Original auf ein Generikum problemlos, abgesehen von seltenen Nebenwirkungen auf Hilfsstoffe. Die Ausnahme bilden die Critical Dose Drugs (Arzneistoffe mit geringer therapeutischer Breite). Bei diesen Substanzen müssen Vor- und Nachteile der Substitution gut geprüft werden. Eine Umstellung auf ein Generikum bedarf einer genauen Überwachung des Patienten. Diese hat auch ihren Preis. Es stellt sich daher im Einzelfall die Frage, ob eine Umstellung ökonomisch und medizinisch angebracht ist. Leider gibt es in der Schweiz meines Wissens bisher noch keine Richtlinien, bei welchen Medikamenten ein Wechsel zum Generikum nicht sinnvoll ist. Solche Richtlinien sollten von einem unabhängigen Fachgremium erstellt werden.

In der Schweiz ist der Anteil der Generika am generikafähigen Markt wegen den oben beschriebenen Widerstände relativ tief. Hilfreich und einfach umzusetzen ist die Wirkstoffrezeptierung bei Spitalaustritt. So entfällt der die Substitution hemmende Ausstattungseffekt (Abgabe von Originalen). Die Steigerung der Motivation zur Akzeptanz der Generika ist sowie Patienten entstehen. Ansonsten würden die diesbezüglichen Aufwendungen den Spareffekt wieder neutralisieren. Der Generikabereich ist ja nur ein kleines Stück vom grossen Gesundheitskostenkuchen: Der generikafähige Markt machte 2010 mit 970 Millionen Franken gerade einmal 1,5\% der Gesamtgesundheitskosten aus [6].

\section{Literatur}

1 Rebecca L. Waber, Baba Shiv, Ziv Cameron, Dan Ariely. Commercial features of placebo and therapeutic efficacy. JAMA. 2008;299(9):1016-7.

2 Kesselheim AS, Misono AS, Lee JL, Stedman MR, Brookhart MA, Choudhry NK et al., Clinical equivalence of generic and brand-name drugs used in Cardiovascular disease: a systematic review and meta-analysis. JAMA. 2008;300(21):2514-26. Review.

3 Gregory L. Krauss, Brian Caffo, Yi-Ting Chang, Craig W. Hendrix, Kelly Chuang. Assessing bioequivalence of generic antiepilepsy drugs. Annals of Neurology. 2011; Vol. 70,2:221-8.

4 Rascati KL, Richards KM, Johnsrud MT, Mann TA. Effect of antiepileptic drug substitutions on epileptic events requiring acute care. Pharmacotherapy. 2009;29(7):769-74

5 Kahneman D, Tversky A. Prospect Theory: An Analysis of Decision under Risk. Econometrica. 1979;47:263-91.

6 www.interpharma.ch

7 www.hexal.de

8 IMS, Stand Januar 2010. 